\title{
Complex organic molecules in protostellar environments in the SKA era
}

\author{
C. Codella ${ }^{1}$, L. Podio*1, F. Fontani ${ }^{1}$, I. Jiménez-Serra ${ }^{2}$, P. Caselli ${ }^{3}$, C. Ceccarelli ${ }^{4}$,
} M.E. Palumbo ${ }^{5}$, A. López-Sepulcre ${ }^{6,4}$, M.T. Beltrán ${ }^{1}$, B. Lefloch ${ }^{4}$, J.R. Brucato ${ }^{1}$, S. Viti $^{7}$, L. Testi ${ }^{2,1}$

${ }^{1}$ INAF, Osservatorio Astrofisico di Arcetri, Largo E. Fermi 5, 50125 Firenze (Italy); ${ }^{2}$ ESO, Karl Schwarzschild srt. 2, 85748 Garching (Germany); ${ }^{3}$ MPE, Giessenbachstr.1, 85748 Garching (Germany); ${ }^{4}$ IPAG, UMR 5274, UJF-Grenoble 1/CNRS-INSU, 38041 Grenoble (France); 5 INAF, Osservatorio Astrofisico di Catania, via S. Sofia 78, 95123 Catania (Italy); ${ }^{6}$ Department of Physics, The University of Tokyo, Bunkyo-ku, Tokyo 113-0033, Japan; ${ }^{7}$ UCL, Gower Street, WC1E $6 B$ London (UK)

E-mail: codella@arcetri.astro.it

Molecular complexity builds up at each step of the Sun-like star formation process, starting from simple molecules and ending up in large polyatomic species. Complex organic molecules (COMs; such as methyl formate, $\mathrm{HCOOCH}_{3}$, dymethyl ether, $\mathrm{CH}_{3} \mathrm{OCH}_{3}$, formamide, $\mathrm{NH}_{2} \mathrm{CHO}$, or glycoaldehyde, $\mathrm{HCOCH}_{2} \mathrm{OH}$ ) are formed in all the components of the star formation recipe (e.g. pre-stellar cores, hot-corinos, circumstellar disks, shocks induced by fast jets), due to ice grain mantle sublimation or sputtering as well as gas-phase reactions. Understanding in great detail the involved processes is likely the only way to predict the ultimate molecular complexity reached in the ISM, as the detection of large molecules is increasingly more difficult with the increase of the number of atoms constituting them.

Thanks to the recent spectacular progress of astronomical observations, due to the Herschel (sub$\mathrm{mm}$ and IR), IRAM and SMA (mm and sub-mm), and NRAO (cm) telescopes, an enormous activity is being developed in the field of Astrochemistry, extending from astronomical observatories to chemical laboratories. We are involved in several observational projects providing unbiased spectral surveys (in the 80-300 and 500-2000 GHz ranges) with unprecedented sensitivity of templates of dense cores and protostars. Forests of COM lines have been detected. In this chapter we will focus on the chemistry of both cold prestellar cores and hot shocked regions, (i) reviewing results and open questions provided by mm-FIR observations, and (ii) showing the need of carrying on the observations of COMs at lower frequencies, where SKA will operate. We will also emphasize the importance of analysing the spectra by the light of the experimental studies performed by our team, who is investigating the chemical effects induced by ionising radiation bombarding astrophysically relevant ices.

Advancing Astrophysics with the Square Kilometre Array

June 8-13, 2014

Giardini Naxos, Italy

${ }^{*}$ Speaker. 


\section{The molecular complexity in a Sun-like forming system}

The formation of Sun-like stars and the chemical complexity of the molecular gas involved in the process are sketched in Fig. 1, following Caselli \& Ceccarelli (2012), and here summarised:

1. matter slowly accumulates toward the center of a molecular cloud. The central density increases while the temperature decreases, forming the so-called prestellar cores. Atoms and molecules in the gas phase freeze-out onto the cold surfaces of the dust grains, forming the grain mantles. Hydrogenation of atoms and molecules takes place, forming molecules such as water $\left(\mathrm{H}_{2} \mathrm{O}\right)$, formaldehyde $\left(\mathrm{H}_{2} \mathrm{CO}\right)$ and methanol $\left(\mathrm{CH}_{3} \mathrm{OH}\right)$. In these regions the formation of new molecules in icy mantles is also caused by the effects of $\mathrm{UV}$ photons and low-energy cosmic rays. Indeed, the recent detection of $\mathrm{CH}_{3} \mathrm{CHO}, \mathrm{CH}_{3} \mathrm{OCH}_{3}, \mathrm{CH}_{3} \mathrm{OHO}$, and $\mathrm{CH}_{2} \mathrm{CO}$ in the prestellar core $\mathrm{L} 1689 \mathrm{~B}$ (Bacmann et al. 2012) shows that COM synthesis has already started at the prestellar stage.

2. The collapse starts, the gravitational energy is converted into radiation and the envelope around the central object warms up. The molecules frozen on the mantles acquire mobility and form new, more complex species. When the temperature reaches about $100 \mathrm{~K}$ mantle sublimates, and we have the so called hot corinos $(\leq 0.01 \mathrm{pc})$ phase. Molecules in the mantles are injected in the gas, where they react and form new, more complex, molecules. The abundance of COMs (such as methyl formate, $\mathrm{HCOOCH}_{3}$, or dymethyl ether, $\mathrm{CH}_{3} \mathrm{OCH}_{3}$ ) dramatically increases. A classical example is provided by IRAS16293-2422 (e.g. Cazaux et al. 2003; Ceccarelli et al. 2007; Bottinelli et al. 2007), where recently also glycoaldehyde $\left(\mathrm{HCOCH}_{2} \mathrm{OH}\right)$, crucial molecules for the formation of metabolic molecules, has been detected (Jörgensen et al. 2012).

3. Simultaneously to the collapse, a newborn protostar generates a fast and well collimated jet, possibly surrounded by a wider angle wind. In turn, the ejected material drives shocks travelling through the surrounding high-density medium. Shocks heat the gas up to thousands of $\mathrm{K}$ and trigger several processes such as endothermic chemical reactions and ice grain mantle sublimation or sputtering. Several molecular species undergo significant enhancements in their abundances. The prototypical chemical rich shock is L1157-B1. Towards this source, not only relatively simple complex molecules, like methanol, have been detected (Bachiller et al. 2001), but also molecules considered hot corinos tracers, like methyl cyanide $\left(\mathrm{CH}_{3} \mathrm{CN}\right)$, ethanol $\left(\mathrm{C}_{2} \mathrm{H}_{5} \mathrm{OH}\right)$, formic acid $(\mathrm{HCOOH})$, and $\mathrm{HCOOCH}_{3}$ (Arce et al. 2008). The emission of these species is concentrated in a small (around $1000 \mathrm{AU}$ ) region associated with the violent shocks at the head of the outflowing material (Codella et al. 2009). The presence of COMs in molecular outflows strongly suggests that these species were part of the sputtered icy mantles as the time elapsed since the shock is too short for any gas-phase route to build up COMs.

4. The envelope dissipates with time and eventually only a circumstellar disk remains, also called protoplanetary disk. In the hot regions, close to the central forming star, new complex molecules are synthesized by reactions between the species formed in the protostellar phase. In the cold regions of the disk, where the vast majority of matter resides, the molecules 


\section{FROM A DIFFUSE CLOUD TO A SUN + PLANETARY SYSTEM FROM ATOMS \& SIMPLE MOLECULES TO LIFE}

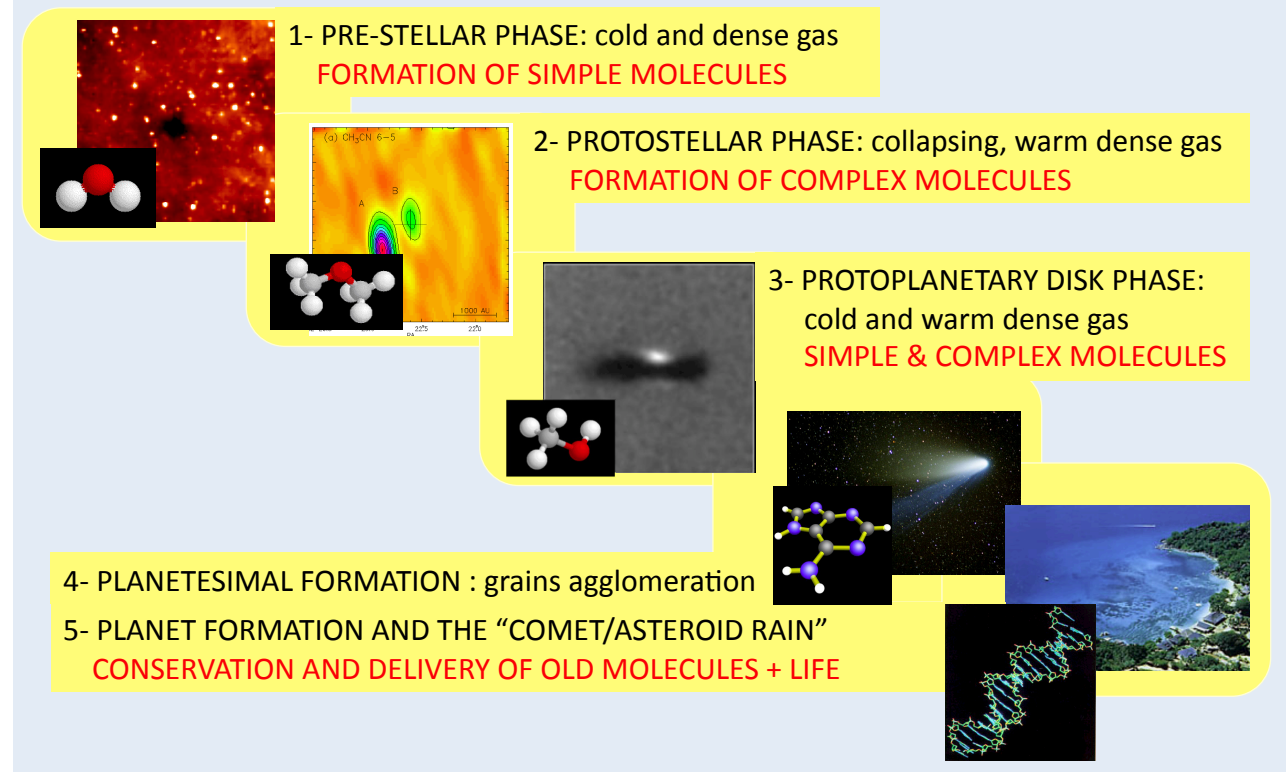

Figure 1: Star formation and chemical complexity (from Caselli \& Ceccarelli 2012). The formation of a star and a planetary system, like the Solar System, passes through different phases, marked in the sketch.

formed in the protostellar phase freeze-out onto the grain mantles again. Dust grains then coagulate into larger planetesimals, the bricks of future planets, comets, and asteroids.

In this chapter, we focus on the earliest stages reviewing the importance of COMs in the prestellar cores as well as in the hot shocked regions produced by protostellar jets. Planetary disks will be discussed in a separate chapter (Testi et al. 2014).

\section{The lessons provided by mm-FIR unbiased spectral surveys}

In the last years several large programs started collecting unbiased spectral surveys with high frequency resolutions and unprecedented sensitivities of different targets considered among the typical laboratories of different stages of the low-mass star forming process (as e.g. prestellar cores, hot-corinos, protostellar shocks, more evolved Class I objects). One of the main goals is indeed the detection of complex and rare molecular species in the interstellar space through emission due to their ro-vibrational transitions. In particular, these efforts have been recently carried out in the 80$300 \mathrm{GHz}$ range using the IRAM 30-m ground based observatory (ASAI: Astrochemical Surveys At IRAM; http://www.oan.es/asai) and between $500 \mathrm{GHz}$ and $2000 \mathrm{GHz}$ using the ESO Herschel Space Observatory (CHESS: Chemical HErschel Surveys of Star forming regions; http://wwwlaog.obs.ujf-grenoble.fr/heberges/chess/). The collected spectra are very rich in COMs, reflecting 

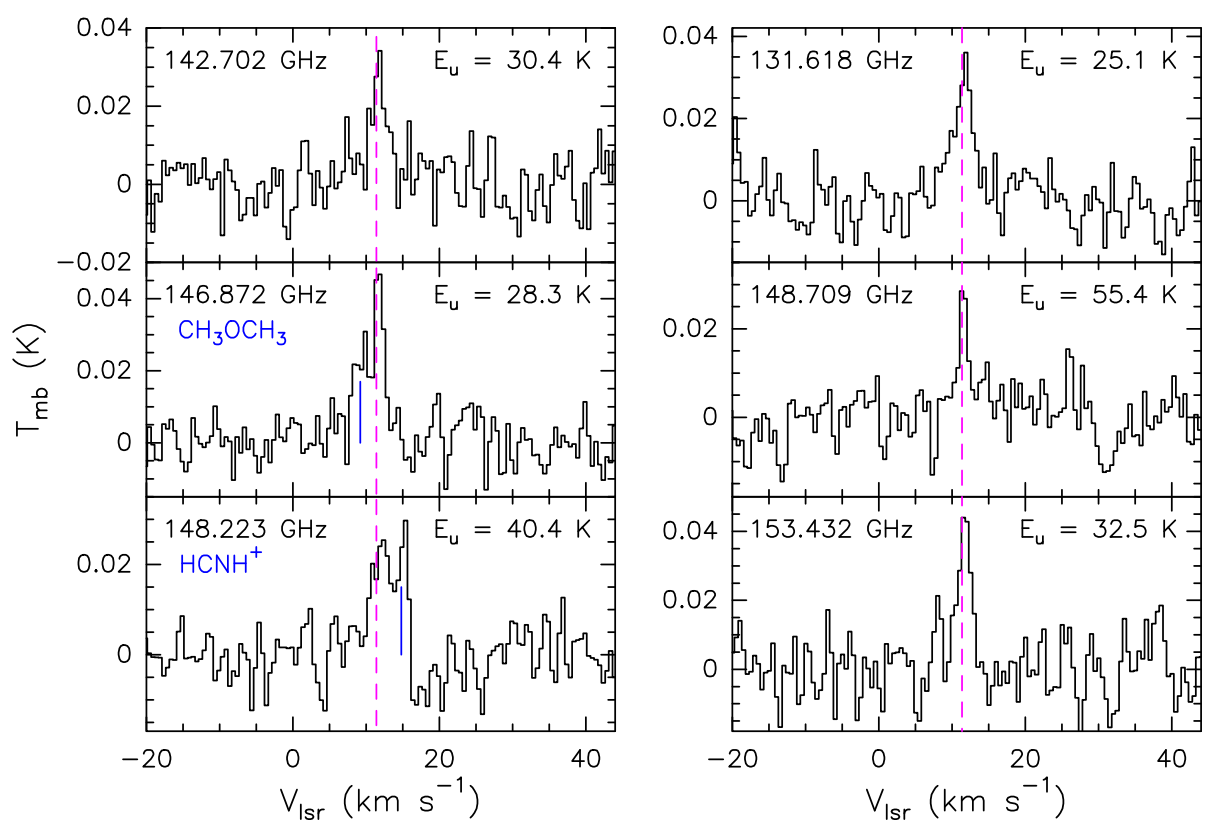

Figure 2: $\mathrm{NH}_{2} \mathrm{CHO}$ lines detected at $2 \mathrm{~mm}$ in OMC-2 FIR4 with the IRAM 30-m antenna in the ASAI framework (from López-Sepulcre et al. 2014). The magenta dashed lines mark the systemic velocity of OMC-2 FIR4. The blue solid lines mark the position of blended lines.

the chemical complexity of all the components involved in the low-mass star formation, and, in the case of hot-corinos around protostars and jet-driven shocks amazing forest of lines are detected (e.g. Ceccarelli et al. 2010). The analysis of the large number of emission lines due to COMs is still in progress, but the preliminary results are very encouraging: for instance formamide $\left(\mathrm{NH}_{2} \mathrm{CHO}\right)$, the simplest possible amide and a central molecule in the synthesis of metabolic and genetic molecules, has been revealed towards IRAS16293-2422, NGC1333-IRAS4A, SVS13A, OMC-2 FIR4, CephE, and the L1157-B1 shock (Kahane et al. 2013; Mendoza et al. 2014; López-Sepulcre et al. 2014, see Fig. 2).

A huge effort is carried out also using interferometers to provide high-spatial resolution images. The IRAM Plateau de Bure interferometer (PdBI) large program CALYPSO (Continuum and Lines from Young ProtoStellar Objects; http://irfu.cea.fr/Projects/Calypso) is providing the first sub-arcsecond statistical study, in the $80-300 \mathrm{GHz}$ window, of the inner environments of the low-luminosity Class 0 sources. Also in this case spectacular forests of lines are observed, showing an amazing large number of lines at high excitation due to COMs such as ethylene glycol $\left(a \mathrm{Ga}^{\prime}-\right.$ $\left.\left(\mathrm{CH}_{2} \mathrm{OH}\right)_{2}\right)$ and $\mathrm{NH}_{2} \mathrm{CHO}$. As an example, Fig. 3 shows the NGC1333-IRAS2A case (Maury et al. 2014): COM emission inside the protostellar envelope has been imaged, finding that it originates from a region of radius $40-100 \mathrm{AU}$, centered on the protostar. This spatial scale is consistent with the size of the inner envelope where $T_{\text {dust }} \geq 100 \mathrm{~K}$ is expected, supporting the hot-corino origin.

In conclusion, the synergy between spectral surveys using single-dishes and interferometric observations is of paramount importance to detect and analyse the COM emission. On the one hand, the unbiased spectral surveys provide the largest possible frequency range, and thus the most complete census of COMs; a multiline approach is also needed to safely identify the emission 


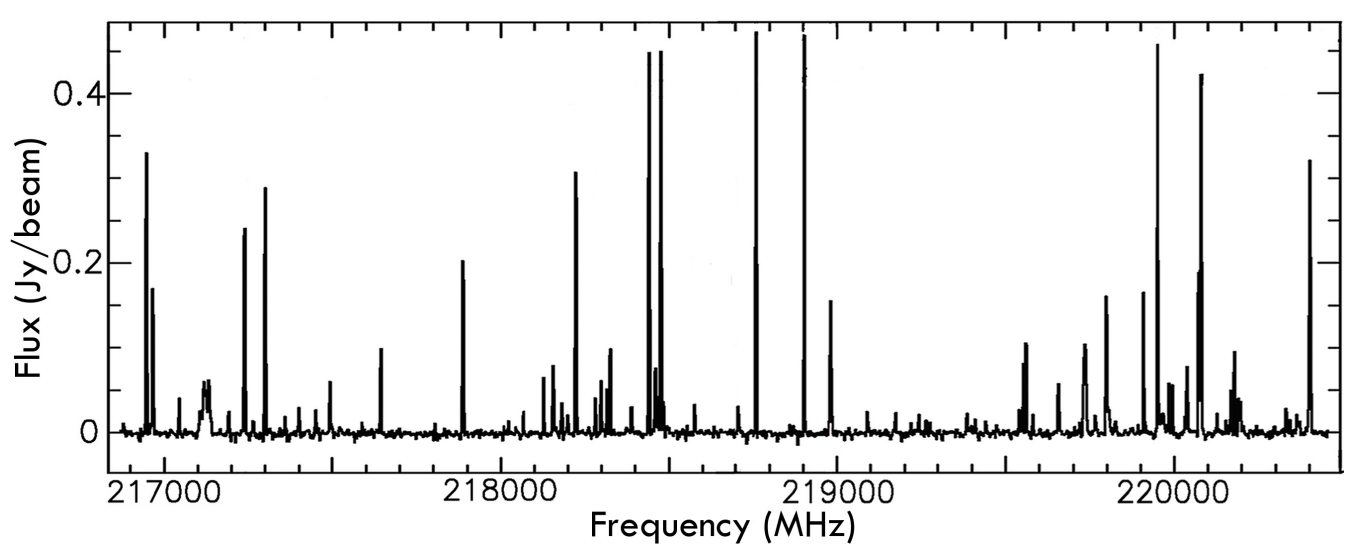

Figure 3: Continuum-subtracted PdBI spectrum around $218.5 \mathrm{GHz}$, at the position of the IRAS2A protostar (from Maury et al. 2014). Several COMs are detected, as e.g. $\mathrm{CH}_{3} \mathrm{OCH}_{3}, \mathrm{CH}_{3} \mathrm{OCHO}, a G a^{\prime}-\left(\mathrm{CH}_{2} \mathrm{OH}\right)_{2}$, and $\mathrm{NH}_{2} \mathrm{CHO}$.

spectrum of complex species. On the other hand, interferometric images provide the size and morphology of the emitting regions, overcome the filling factor limitation of single-dish spectra, and allow one to propely derive physical conditions such as density, temperature, and abundance. In addition, the linewidths of COMs are typically reduced in interferometric observations, which allows a better identification of the molecular lines since it reduces line blending.

\section{Cold cases: prestellar cores and very early protostars}

\subsection{Detectability of aminoacids in Solar-system precursors}

The increasing performance of millimeter instrumentation in the past years has opened up the possibility to carry out high-sensitivity molecular line surveys even toward the earliest (and coldest) stages of low-mass star formation. These initial conditions are represented by cold dark cores and in particular, by pre-stellar cores, i.e. dense and cold condensations on the verge of gravitational collapse (central $\mathrm{H}_{2}$ densities of some $10^{4} \mathrm{~cm}^{-3}$ and temperatures $\leq 10 \mathrm{~K}$; Caselli et al. 1999; Crapsi et al. 2007). These observations have shown that $\mathrm{COMs}$ (such as propylene $-\mathrm{CH}_{2} \mathrm{CHCH}_{3}$ or acetaldehyde $-\mathrm{CH}_{3} \mathrm{CHO}$ ) are also present in the cold gas of these cores, unexpectedly revealing a high chemical complexity in these objects (see Marcelino et al. 2007 and Bacmann et al. 2012).

Among these complex organics, amino acids are of high interest in Astrochemistry and Astrobiology because of their role in the synthesis of proteins in living organisms. It is currently believed that their formation may have occurred in the interstellar medium (ISM) since amino acids, including glycine and alanine, have been found in meteorites (Pizzarello et al. 1991; Ehrenfreund et al. 2001; Glavin et al. 2006) and comets (as in Wild 2; Elsila et al 2009). This idea is supported by laboratory experiments which have shown that amino acids precursor are largely produced in UV-photon and ion-irradiated interstellar ice analogs (Munõz-Caro et al. 2002; Bernstein et al. 2002; Holtom et al. 2005). The detection of amino acids in the ISM is however challenging since their partition function is large and therefore, their emission is spread among many transitions. The detection of amino acids in the ISM remains to be reported. 
Recently, it has been proposed that pre-stellar cores may be well suited for the detection of amino acids in the ISM (Jiménez-Serra et al. 2014). The gas temperatures in pre-stellar cores are $\leq 10 \mathrm{~K}$, which yields a low level of line confusion since the number of molecular transitions excited at these temperatures is small. The linewidths of the molecular line emission in these cores are $\leq 0.5 \mathrm{~km} \mathrm{~s}^{-1}$, which allows accurate identifications of the observed molecular lines because they suffer less from line blending. In addition, water vapour has recently been found toward the central few thousand AU of one pre-stellar core, L1544, which indicates that a small fraction of the ices ( $\sim 0.5 \%$ of the total water abundance in the mantles) has been released into the gas phase (Caselli et al. 2012). These authors have proposed that water vapour in L1544 is produced by the partial photo-desorption of ices by secondary, cosmic ray induced UV-photons. Since COMs form in the outer layers of the mantles (Cuppen et al. 2009; Taquet et al. 2012), these molecules are also expected to be photo-desorbed together with water in pre-stellar cores, making these objects excellent candidates to test the detectability of amino acids in Solar-system precursors.

Simple radiative transfer calculations of glycine $\left(\mathrm{NH}_{2} \mathrm{CH}_{2} \mathrm{COOH}\right.$, i.e. the simplest amino acid) towards the L1544 pre-stellar core show that several glycine lines could reach detectable levels (peak line intensities $\geq 10 \mathrm{mK}$ ) in the frequency range between 60 and $80 \mathrm{GHz}$ (Jiménez Serra et al. 2014). We have extrapolated these results to the frequency range covered by SKA1MID Bands 4 and 5, where we evaluate whether glycine could be detected with this instrument (see Fig. 4). These calculations assume a solid abundance of glycine in ices of a few $10^{-4}$ with respect to water, similar to those synthesized in laboratory experiments of UV photon- and ion-irradiated interstellar ice analogs (Munõz-Caro et al. 2002; Bernstein et al. 2002; Holtom et al. 2005). This solid abundance translates into a maximum gas-phase abundance of glycine of $\sim 8 \times 10^{-11}$ after ice photo-desorption.

In Fig. 4, we report the predicted intensities of glycine for the L1544 core, assuming that the gas-phase abundance of glycine (of $\sim 8 \times 10^{-11}$ ) remains constant across the core. The velocity resolution used in the simulations is $\sim 0.2 \mathrm{~km} \mathrm{~s}^{-1}$ and the expected linewidth of the glycine emission is $\sim 0.3 \mathrm{~km} \mathrm{~s}^{-1}$. The assumed source size is $\sim 12$ ". Figure 4 shows that a few glycine lines could reach detectable levels with $\mathrm{S} / \mathrm{N}$ ratios $\geq 3$ in a total of $\sim 1000 \mathrm{hrs}$ of integration time in SKA1-MID Band 5. For this integration time, a rms noise level of 50 microJy is indeed expected at a velocity resolution of $0.2 \mathrm{~km} \mathrm{~s}^{-1}$ within a 12"-beam (see the Level 0 Science Requirement SKA1-SCI-19). However, we stress that if the frequency coverage of SKA were extended to higher frequencies (e.g. up to $25 \mathrm{GHz}$, which would also allow to observe the $\mathrm{NH}_{3}(1,1)$ inversion lines at $\sim 23 \mathrm{GHz}$; see Fig. 4), this detection experiment could be performed in 10 times less time thanks to the presence of 3 times brighter glycine lines around $\sim 20 \mathrm{GHz}$ (intensities $\geq 0.5 \mathrm{mJy} \mathrm{beam}^{-1}$ ). In any case, a gas-phase abundance as low as $\sim 8 \times 10^{-11}$ would be the lowest abundance of glycine that could be detected with this experiment in SKA1-MID Band 5.

It is important to stress that Bands 1 and 2 of ALMA are currently unfunded, and therefore SKA may represent in the future the only instrument that could perform this kind of detection experiment for amino acids. We also note that although glycine shows a collection of transitions at millimetre wavelengths, observations at centimetre wavelengths with SKA1-MID Band 5 (8.8 to $13.8 \mathrm{GHz}$ ) are essential to provide reliable identifications of these lines due to the increasing frequency span between transitions at longer wavelengths (preventing line blending and line confusion). In addition, other complex organics such as the precursors of the glycine chemistry, e.g. 


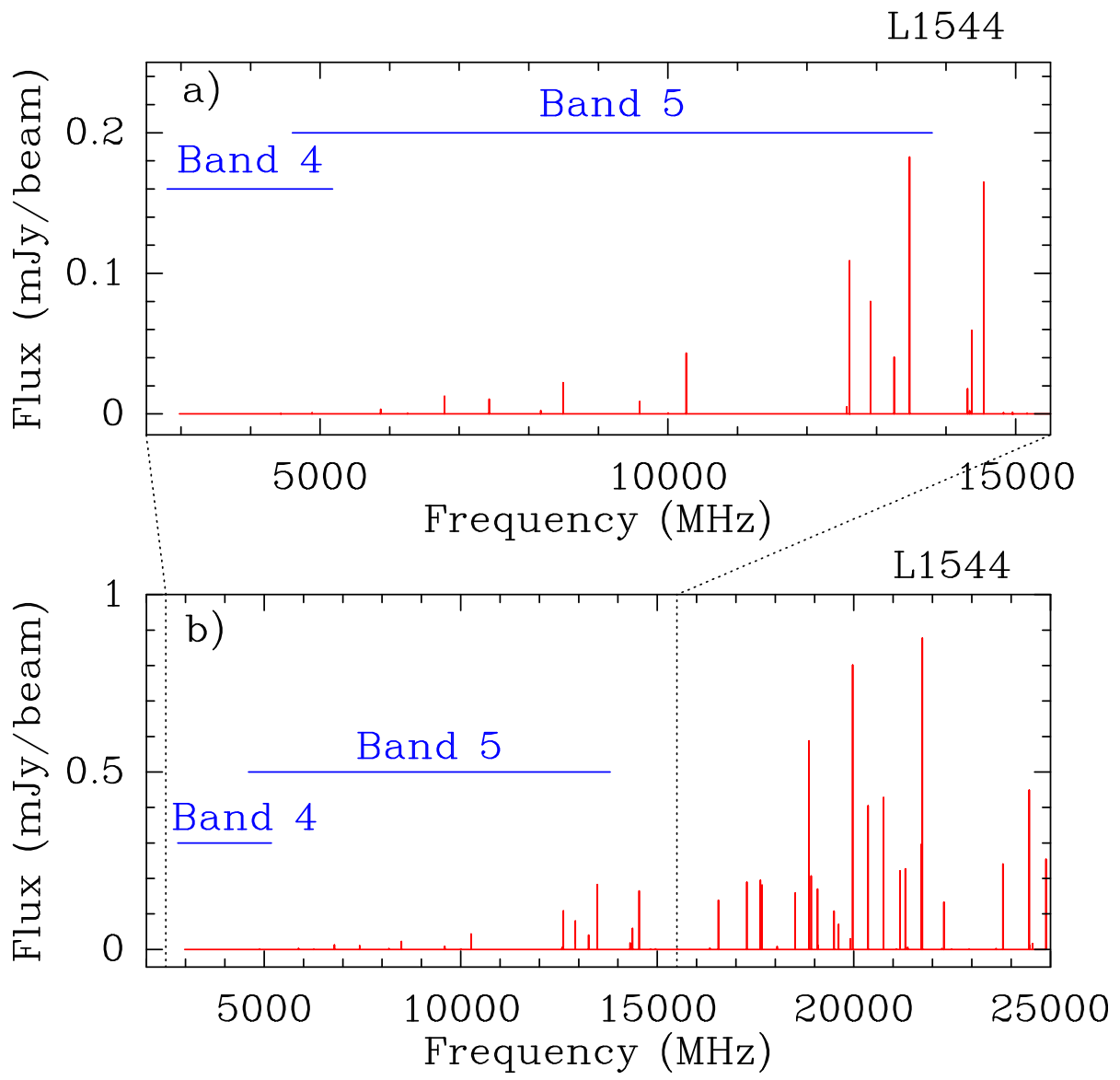

Figure 4: Upper panel: Simulations of the spectrum of glycine (conformer I) obtained for the frequency range between $2.5 \mathrm{GHz}$ and $15.5 \mathrm{GHz}$, considering the physical structure of the $\mathrm{L} 1544$ pre-stellar core (Caselli et al. 2012), a solid glycine abundance of a few $10^{-4}$ with respect to water in ices, and LTE conditions. Horizontal blue lines indicate the frequency coverage of SKA1-MID Bands 4 and 5. Lower panel: LTE spectrum of glycine predicted for frequencies between $2.5 \mathrm{GHz}$ and $25 \mathrm{GHz}$. The glycine lines around $20 \mathrm{GHz}$ are factors of $\sim 3$ brighter than those covered by SKA1-MID Band 5, which would allow the detection of this amino acid in 10 times less observing time.

amino acetonitrile or methyl formate, are expected to be factors of $\sim 100$ more abundant than glycine (Belloche et al. 2008, 2013), and therefore they would be easier to detect with SKA1-MID Bands 4 and 5.

The detection of glycine, and of its precursors, in these Solar-system precursors will represent a major milestone in Astrochemistry and Astrobiology, providing a unique opportunity to link the pre-biotic chemistry in the ISM to their subsequent delivery onto planetary systems.

\subsection{Deuterated COMs}

There are no doubts that one of the most important chemical processes occuring in the cold $(\mathrm{T} \leq 20 \mathrm{~K})$ and dense $\left(\geq 10^{4} \mathrm{~cm}^{-3}\right)$ pre-stellar cores is the freeze-out of atoms and molecules on the surface of dust grains, where the depletion of both C-bearing and N-bearing molecules is found to be high in low- and high-mass pre-stellar cores (e.g. Caselli et al. 1999, Friesen et al. 2010, 
Fontani et al. 2012). Frozen on grain mantles, atoms and molecules can undergo hydrogenation, due to the high mobility of the light $\mathrm{H}$ atoms. In particular, from hydrogenation of $\mathrm{CO}$ (the most abundant neutral molecule after $\mathrm{H}_{2}$ ) one forms sequentially $\mathrm{HCO}, \mathrm{H}_{2} \mathrm{CO}$ and $\mathrm{CH}_{3} \mathrm{OH}$, which is thus the species that is formed last. Moreover, the low temperature favours the deuteration of the species mentioned, because of the endothermicity of the chemical reactions which replace $\mathrm{D}$ atoms into their hydrogenated counterparts. Therefore, as time proceeds, the formation of methanol and its deuterated forms $\left(\mathrm{CH}_{2} \mathrm{DOH}, \mathrm{CH}_{3} \mathrm{OD}, \mathrm{CHD}_{2} \mathrm{OH}\right.$, etc. $)$ is boosted, until the energy released by the nascent protostellar object in the form of radiation increases the temperature of its environment, causing the evaporation of the grain mantles and the release of these molecules into the gas. As the temperature increases, the deuterated species are expected to get gradually destroyed due to the higher efficiency of the backward endothermic reactions (Caselli \& Ceccarelli 2012). Therefore, high deuterated fractions of methanol, i.e. the ratio between the column density of the species containing $\mathrm{D}$ and that of $\mathrm{CH}_{3} \mathrm{OH}$, are expected to be powerful tracers of the short-living evolutionary stage in between the pre-stellar and the protostellar phase, at which the evaporation/sputtering of the grain mantles is efficient, and the warm gas-phase reactions have no time to change significantly the chemical composition of the gas.

Of course, testing these predictions is challenging because the deuterated forms of methanol are supposed to originate faint lines, which, at (sub-)millimeter wavelengths, can be easily overwhelmed by nearby stronger emission lines of lighter and more abundant molecules. Several transitions of $\mathrm{CH}_{2} \mathrm{DOH}$ can be observed in the range $1-15 \mathrm{GHz}$ as can be seen in Fig. 5. In this spectral window, the contamination of lines of more abundant molecules is expected to be negligible. The combination of high angular resolution and high sensitivity offered by SKA (see Sect. 7) will be eminently suitable to map these lines, which are expected to arise from very compact regions (sizes $\leq 3$ ", e.g. Peng et al. 2012). As an example, we can estimate the time required to detected some of the lines shown in the synthetic spectrum in Fig. 5. The spectrum includes all transitions of $\mathrm{CH}_{2} \mathrm{DOH}$ in the spectroscopic band $\sim 1-15 \mathrm{GHz}$, as modeled by GILDAS-Weeds package (Maret et al. 2011) assuming the following parameters: $T_{\text {kin }}=20 \mathrm{~K}, N\left(\mathrm{CH}_{2} \mathrm{DOH}\right)=5 \times 10^{15} \mathrm{~cm}^{-2}$, source size $=1 "$, line FWHM $=1 \mathrm{~km} \mathrm{~s}^{-1}$. The column density assumed is a mean source-averaged value measured towards protostellar cores (e.g. Parise et al. 2006; Fontani et al. 2014b). Using the SKA1 Imaging Science Performance document (Braun R., 2014-06-02 version), at a rest frequency of $5 \mathrm{GHz}$, assuming $T_{\mathrm{sys}}=20 \mathrm{~K}, 190$ dishes of $15 \mathrm{~m}, 2$ polarisations, a velocity resolution of $\sim 1 \mathrm{~km}$ $\mathrm{s}^{-1}$, the $1 \sigma \mathrm{rms}$ noise in the spectrum after $50 \mathrm{hrs}$ of integration on source is $0.077 \mathrm{mJy}$. Figure 5 tells us that several lines have intensity peak well above $3 \sigma$. Therefore, this will allow us not only to just detect the presence of the molecule, but also to derive estimates of some important physical parameters (e.g. rotation temperature and column density from the rotation diagrams).

Moreover, other deuterated species of complex molecules $\left(\mathrm{CH}_{2} \mathrm{DCCH}, \mathrm{HDNCH}_{2} \mathrm{CN}\right.$, etc.) possess lines in the same frequency range. If detected, these species will put other relevant constraints on chemical models, in an effort to reach a general knowledge of the deuteration mechanism(s) during the earliest phases of the star formation process.

\section{Hot protostellar shocks versus hot-corinos}

The L1157 region at 250 pc hosts a Class 0 protostar (L1157-mm) driving a spectacular chem- 


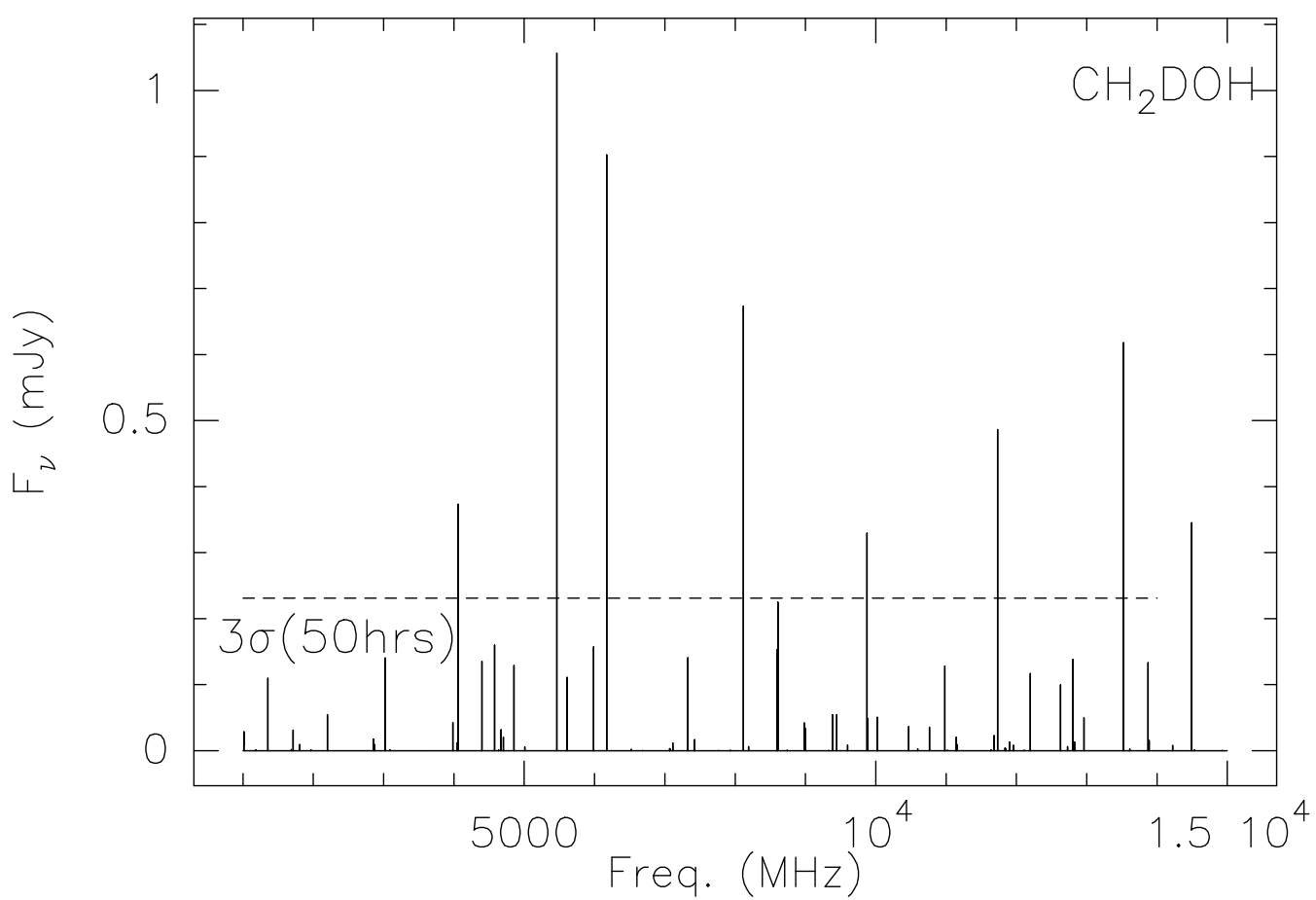

Figure 5: Synthetic spectrum of $\mathrm{CH}_{2} \mathrm{DOH}$ modeled for SKA1-MID with GILDAS-Weeds package (Maret et al. 2011) in the range $\sim 1-15 \mathrm{GHz}$, assuming LTE conditions, $T_{\text {kin }}=20 \mathrm{~K}, N\left(\mathrm{CH}_{2} \mathrm{DOH}\right)=5 \times 10^{15}$ $\mathrm{cm}^{-2}$, source size $=1 "$, and line FWHM $=1 \mathrm{~km} \mathrm{~s}^{-1}$. The dashed line represents the expected $3 \sigma$ level in the spectrum that can be achieved after 50 hours of integration on source (see text for details).

ically rich bipolar outflow (Bachiller et al. 2001). The southern lobe is associated with two cavities seen in the IR $\mathrm{H}_{2}$ and CO lines (e.g. Neufeld et al. 2009; Gueth et al. 1996), likely created by episodic events in a precessing jet. Located at the apex of the more recent cavity, the bright bow shock called B1 has a kinematical age of 2000 years. The bow shocks, when mapped with the IRAM PdB and VLA interferometers, reveal a clumpy structure, with clumps located at the wall of the cavity (e.g. Tafalla \& Bachiller 1995; Codella et al. 2009), and well traced by typical products of both grain mantle sputtering (such as $\mathrm{NH}_{3}, \mathrm{CH}_{3} \mathrm{OH}$, and $\mathrm{H}_{2} \mathrm{CO}$ ) and refractory core disruption (SiO). Thus, the young L1157-B1 shock offers an exceptional opportunity to investigate in details the chemical composition of the grain ice mantles as well as how the gas phase is chemically enriched after the shock occurrence.

Thanks to PdBI observations (see Fig. 6), three COMs have been so far imaged in L1157B1: $\mathrm{CH}_{3} \mathrm{OH}$, acetaldehyde $\left(\mathrm{CH}_{3} \mathrm{CHO}\right)$, and $\mathrm{CH}_{3} \mathrm{CN}$ (Benedettini et al. 2007, 2013; Codella et al. 2009 , 2014). The $\mathrm{CH}_{3} \mathrm{CN}$ image shows a clumpy structure superimposed to the classical B1 archlike shape, displaying a unique continuous structure tracing the propagation of a large bow shock. In principle, $\mathrm{CH}_{3} \mathrm{CN}$ could form on the surface of grains and then injected in the gas phase due to shock sputtering. Alternatively, $\mathrm{CH}_{3} \mathrm{CN}$ may belong to the so-called second generation species, i.e. the molecules formed in the warm gas-phase chemically enriched after the mantle release (see e.g. Bottinelli et al. 2007; Bisschop et al. 2008). Both scenarios need an increase of temperature, as in the L1157-B1 case (up to about $10^{3} \mathrm{~K}$, Busquet et al. 2013). The $\mathrm{CH}_{3} \mathrm{CN}$-to- $\mathrm{CH}_{3} \mathrm{OH}$ ratio is $\simeq 10^{-4}-10^{-3}$, i.e. smaller with respect to those found in hot-corinos $\left(10^{-2}-10^{-1}\right)$. This difference 


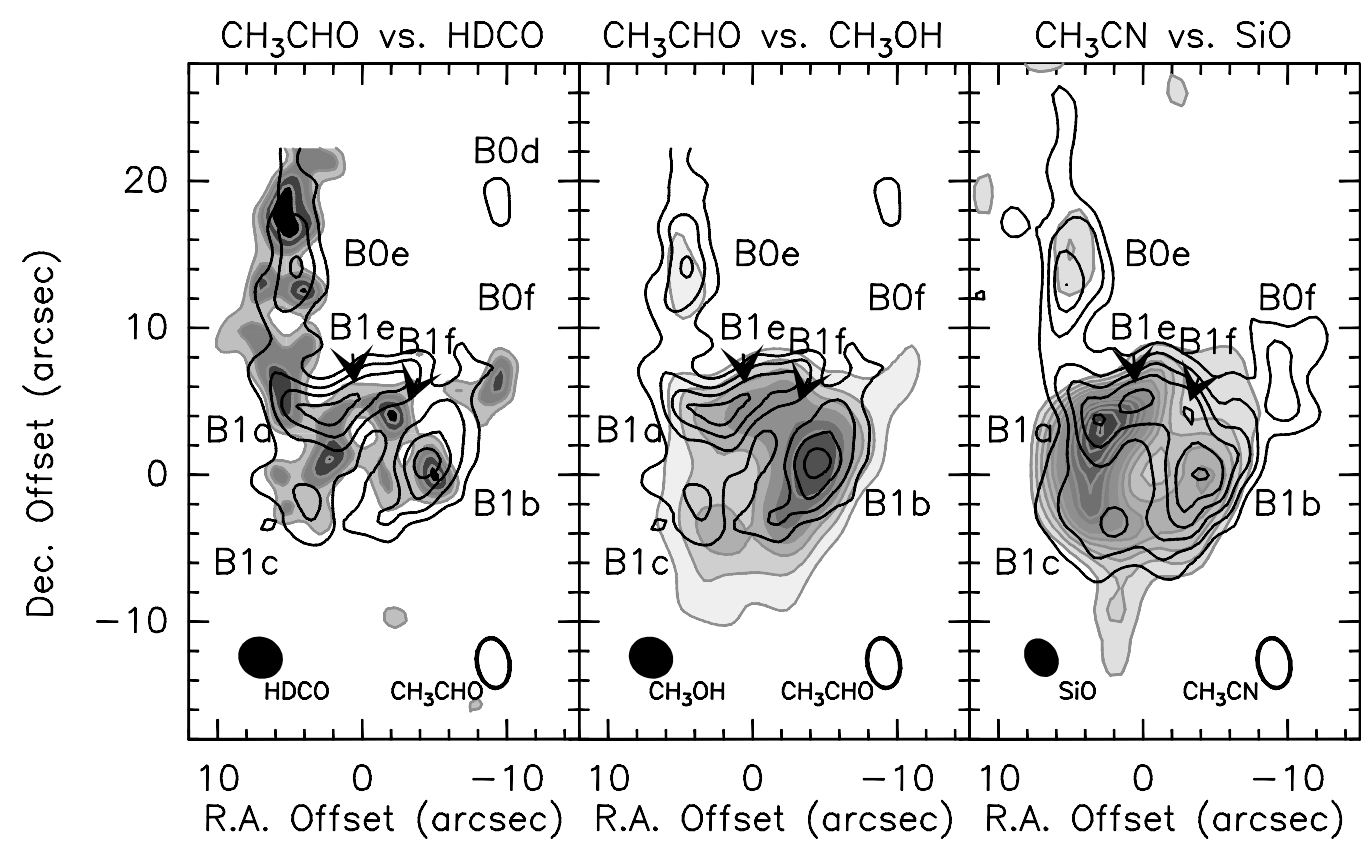

Figure 6: Chemical differentiation in L1157-B1: the maps are centred at $\Delta \alpha=+21^{\circ}$ and $\Delta \delta=-64^{\circ}$ from the driving protostar L1157-mm. Overlay of the integrated intensity IRAM PdBI map at $2 \mathrm{~mm}$ of the $\mathrm{CH}_{3} \mathrm{CHO}\left(7_{0,7}-6_{0,6}\right) \mathrm{AE}$ (contours, from Codella et al. 2014) on the $\mathrm{HDCO}\left(2_{1,1}-1_{0,1}\right)$ (left panel, from Fontani et al. 2014a) and $\mathrm{CH}_{3} \mathrm{OH}\left(3_{K}-2_{K}\right)$ emission (middle panel; from Benedettini et al. 2013). Righ panel compares the $\mathrm{CH}_{3} \mathrm{CN}\left(8_{K}-7_{K}\right)$ and $\mathrm{SiO}(2-1)$ spatial distributions (Gueth et al. 1998, Codella et al. 2009). Labels are for the different L1157-B1 molecular clumps well imaged in the $\mathrm{CH}_{3} \mathrm{CN}$ map. The synthesised beams are shown in the bottom part of the panels.

suggests that a significant amount of $\mathrm{CH}_{3} \mathrm{CN}$ may form in the gas phase and that in L1157-B1 the $\mathrm{CH}_{3} \mathrm{CN}$ abundance has not yet reached its maximum, given the young age of the shock.

Recently, also $\mathrm{CH}_{3} \mathrm{CHO}$ has been mapped at PdBI (Codella et al. 2014), and also in this case there is a good agreement with the $\mathrm{CH}_{3} \mathrm{OH}$ (and $\mathrm{CH}_{3} \mathrm{CN}$ ) spatial distribution, confirming COMs are emitting where mantles have been recently sputtered. This is further indicated by the excellent spatial correpondance with HDCO emission (Fontani et al. 2014a) tracing deuterated formaldehyde formed on grains and then injected in the shocked gas. For acetaldehyde, the abundance ratio with respect to methanol is quite high $\left(\sim 10^{-2}\right)$ and in any case similar to that observed towards a typical hot-corino such as IRAS16293-2422 (Bisschop et al. 2008). These findings support for $\mathrm{CH}_{3} \mathrm{CHO}$ either a direct formation on grain mantles or a quick $(\leq 2000 \mathrm{yr})$ formation in a chemically enriched gas phase.

These first results show the importance of studying shocked regions as laboratories where (i) to investigate the COMs formation routes and (ii) to verify whether the study of hot-corinos chemistry can be affected by COM emission arising from shocked envelope material at the base of the inner (unresolved) jet. Interferometric observations are instructive to minimise beam dilution effects. In addition, the multiline approach is needed to carefully sample the excitation conditions and correctly derive the abundances of COMs. In particular, the 1-15 GHz frequency range allows one to well sample low-excitation $\left(\mathrm{E}_{u} \leq 20 \mathrm{~K}\right)$ and bright $\left(\mathrm{S}^{2} \geq 1 \mathrm{D}^{2}\right)$ emissions due to COMs (see Sect. 7). 


\section{The SKA case for high-mass star formation}

While the detection and study of COMs in low-mass star forming regions has a clear impact on our understaning of the origin of life, we should underline that COMs in massive star forming regions have recently proved to be ideal tracers of the physical conditions of the very central compact core where the massive star forms. In this subsection we therefore briefly make a case for why SKA is the ideal instrument to study COMS in high-mass star forming regions. The earliest stages of the massive star formation are characterised by the occurrence of the so called hot cores, i.e. hot $(\geq 100 \mathrm{~K}$ ), small ( $\leq 0.1 \mathrm{pc}$ ), and massive (up to thousands of solar masses) clumps warmed by the stellar radiation. In fact, the largest body of detections of COMs comes from observations of hot cores. In the standard paradigm, at densities as high as those of hot cores, gases freeze onto the surface of dust grains in the cold quiescent phase of the molecular cloud. This material is processed to greater molecular complexity via a number of possible surface reactions, perhaps catalyzed by UV, cosmic rays or thermal effects, and is subsequently released back into the gas phase as a result of thermal sublimation due to the heating effect of the newly-formed star. The rich chemistry, comprising of species such as ethyl cyanide, glycoaldehyde and its isomers (e.g. Beltrán et al. 2009, Fuente et al. 2014, Calcutt et al. 2014, and references therein), that results from this sublimation, thus potentially provide powerful diagnostics of the physical and chemical history of the very central core where the star is born. Interestingly, and somewhat surprisingly, hot cores differ from hot corinos not only for the larger sizes, but also chemically: when normalized to methanol or formaldehyde, hot cores have typically one order of magnitude less abundant COMs (such as $\mathrm{HCOOCH}_{3}$ or $\mathrm{CH}_{3} \mathrm{OCH}_{3}$ ) than hot corinos (e.g. Herbst and van Dishoeck 2009). Such differences are likely due to various factors, such as the composition of the sublimated ices, governed by the past prestellar history. COMs in hot cores are even more of a powerful star formation diagnostics because they are excellent tracers of the very central (often not spatially resolved) part of the core, where the protostar is born; their detection is therefore a confirmation of the high density warm cores and, most importantly, their emission is so compact that they must trace the most central region of the hot core, close to the YSOs. Clearly interferometric observations are essential in order to resolve such small scale structures.

Despite tracing such a small extent of gas, it is found (e.g. Calcutt et al. 2014) that there are significant variations in abundances and abundance ratios across COMs, making these species also excellent tracers of chemical and physical differentiation across the different classes of objects. Of particular interest in the study of COMs are the three isomers of $\mathrm{C}_{2} \mathrm{H}_{4} \mathrm{O}_{2}$, all of which have now been detected in star-forming regions. Acetic acid and glycolaldehyde are particularly interesting due to their significant prebiotic significance, since the former is linked to the formation of ribose and the latter is only an amine group $\left(\mathrm{NH}_{2}\right)$ away from glycine, the simplest amino acid. However, so far the identification and characerization of COMs in hot cores have been hampered by the incredibly high number of lines in the sub-millimeter spectra: the latter suffer from strong line confusion due to (i) the richness of the spectrum: the higher the gas density and temperature, the more crowded the spectra will be; (ii) blending due to also the large linewidths, and (iii) uncertainties in the laboratory rest frequencies as well as in the observations. These issues lead, in some cases, to only tentative detections and contribute to the scarcity of observed transitions and number of objects. 
The SKA frequency range offers a unique opportunity to identify and study a large number of COMs in high mass star forming regions: the frequency range covered is clear of contamination because the low energy of smaller molecules fall at higher frequencies, hence line confusion is minimized. Interferometric observations are very useful in hot cores since they filter out much of the extended envelope and therefore the linewidths of COMs can be significantly reduced allowing a better identification of the lines. Also, COMs are characerized by the fact that their level population covers many energy levels because of their large partition functions and hence several of their transitions fall in the SKA range from $4 \mathrm{GHz}$ to $\sim 25 \mathrm{GHz}$. As an example, within the $4 \mathrm{GHz}$ limit, there are tens of glycolaldehyde and ethylene glycol transitions covering rotational transitions from $J$ as low as 1 (for ethylene glycol) and 4 (for glycolaldehyde) up to $J=30$. Clearly, by removing the problem of line confusing and simultaneously observing a large number of transitions for each COM we will be able to accurately derive column densities, and hence, with the aid of state of the art chemical and radiative transfer modelling, determine the physical conditions of the most compact cores in high-mass star forming regions. The combination of all these requirements are only met by the SKA.

\section{The need of laboratory experiments}

In dense molecular clouds $\left(n>10^{4} \mathrm{~cm}^{-3}\right)$ gas phase species condense on silicatic and carbonaceous grains giving rise to icy mantles. These mantles are made of molecules which directly freeze out from the gas phase (such as $\mathrm{CO}$ ) and molecules which are formed after grain surface reactions (such as $\mathrm{H}_{2} \mathrm{O}$ ). The presence of icy grain mantles is indirectly deduced from depletion of gas phase species and is observed in the infrared from absorption features attributed to vibrational modes of solid phase molecules superposed to the background stellar spectrum. Ices have been observed in star forming regions (both low- and high-mass young stellar objects) as well as in quiescent dense clouds. It is widely accepted that icy grain mantles are continuously processed by low-energy cosmic rays, electrons, and UV photons.

Ten different molecular species have been firmly identified in interstellar icy grain mantles (e.g. Gibb et al. 2004). In particular, water $\left(\mathrm{H}_{2} \mathrm{O}\right)$, carbon monoxide $(\mathrm{CO})$, carbon dioxide $\left(\mathrm{CO}_{2}\right)$, methanol $\left(\mathrm{CH}_{3} \mathrm{OH}\right)$, methane $\left(\mathrm{CH}_{4}\right)$, carbonyl sulphide (OCS), formaldehyde $\left(\mathrm{H}_{2} \mathrm{CO}\right)$, formic acid $(\mathrm{HCOOH})$, cyanate ion $\left(\mathrm{OCN}^{-}\right)$and ammonia $\left(\mathrm{NH}_{3}\right)$.

Most of the knowledge on the physical and chemical properties of ices is based on the comparison between observations and laboratory experiments performed at low temperature (10-80 K). Laboratory infrared spectra of icy samples show that the profile of absorption bands depends on different parameters such as the other molecules each species is mixed with and the temperature (e.g. Sandford et al. 1988; Ehrenfreund et al. 1999; Palumbo \& Baratta 2000; Öberg et al. 2007). The properties of icy samples also depend on processing by fast ions, electrons, and UV photons (e.g. Grim \& Greenberg 1987; Palumbo \& Strazzulla 1993; Hudson et al. 2001; Strazzulla et al. 2001; Baratta et al. 2002; Bennet et al. 2007; Öberg et al. 2009; Islam et al. 2014). Energetic ions (keV-MeV) passing through molecular solids release energy to the target along the ion track. As a consequence molecular bonds are broken and radicals and molecular fragments recombine giving rise to molecular species not present in the original sample. Furthermore the structure and morphology of the sample is also modified (e.g. Palumbo 2006; Raut et al. 2001). In the case 


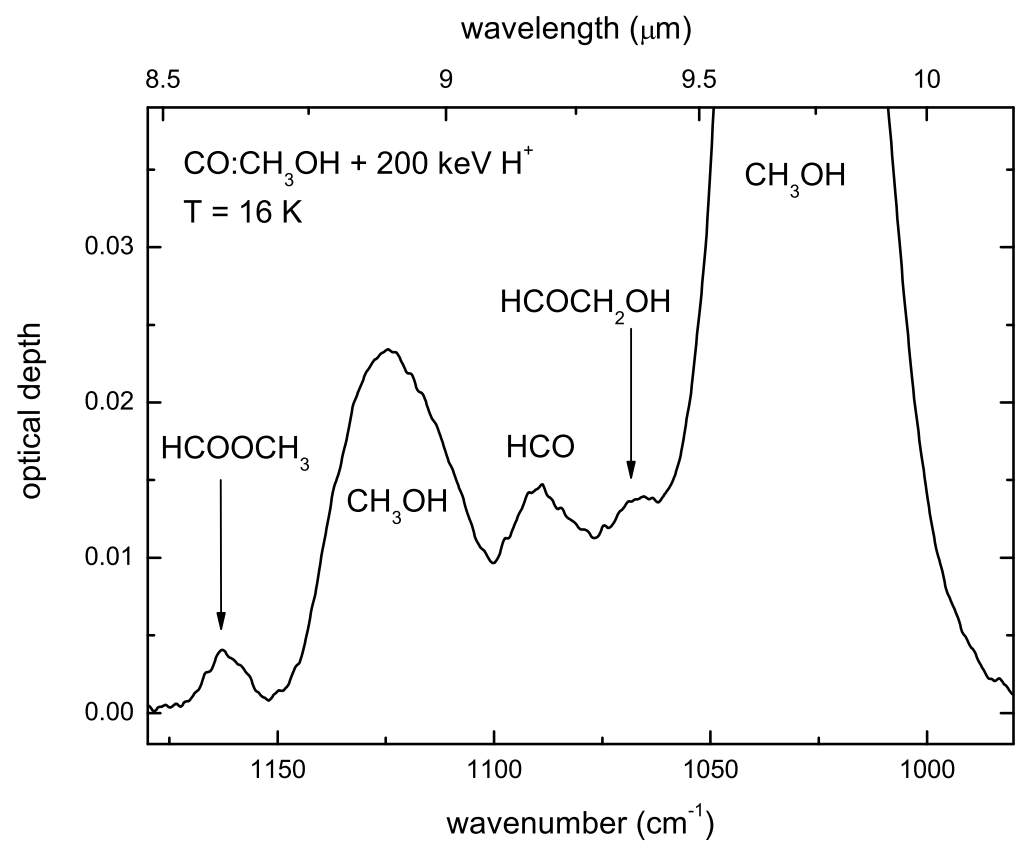

Figure 7: Infrared transmission spectrum (from Modica \& Palumbo 2010), in optical depth scale, of a mixture $\mathrm{CO}: \mathrm{CH}_{3} \mathrm{OH}$ bombarded with $200 \mathrm{keV}$ protons at $16 \mathrm{~K}$. Labels indicate absorption bands due to pristine methanol and bands due to species formed after energetic processing.

of UV photolysis the energy is released to the target material through single photo-dissociation or ionization events per incoming photon. Thus after ion bombardment and UV photolysis the chemical composition and the structure of the sample is modified. Both more volatile and less volatile species are formed and if C-bearing species are present in the original sample a refractory residue is left over after warm-up at room temperature (e.g. Moore et al. 1983; Foti et al. 1984; Palumbo et al. 2004).

In the past 35 years several experiments have been performed to study the effects of ion bombardment and UV photolysis on the chemical composition of icy samples. As an example, laboratory experiments have shown that after energetic processing of methanol the column density of pristine methanol decreases and new bands appear in the infrared spectra indicating the formation of other, also complex, molecules. Species clearly identified are carbon monoxide (CO), carbon dioxide $\left(\mathrm{CO}_{2}\right)$, methane $\left(\mathrm{CH}_{4}\right)$, formyl radical $(\mathrm{HCO})$, formaldehyde $\left(\mathrm{H}_{2} \mathrm{CO}\right)$, ethylene glycol $\left(\mathrm{C}_{2} \mathrm{H}_{4}(\mathrm{OH})_{2}\right)$, methyl formate $\left(\mathrm{HCOOCH}_{3}\right)$, and glycolaldehyde $\left(\mathrm{HCOCH}_{2} \mathrm{OH}\right)($ e.g. Moore et al. 1996; Palumbo et al. 1999, Hudson \& Moore 2000; Bennett et al. 2007; Öberg et al. 2009; Modica \& Palumbo 2010). Figure 7 shows the infrared spectrum in the $1180-980 \mathrm{~cm}^{-1}(8.47-10.20$ $\mu \mathrm{m}$ ) range of a mixture $\mathrm{CO}: \mathrm{CH}_{3} \mathrm{OH}$ bombarded with $200 \mathrm{keV}$ protons at $16 \mathrm{~K}$ (Modica \& Palumbo 2010).

Recently, Compagnini et al. (2009) and Puglisi et al. (2014) have shown, by infrared and Raman spectroscopy, that polyynes $\left(-(\mathrm{C} \equiv \mathrm{C})_{n^{-}}\right)$and polycumulenes $\left(=(\mathrm{C}=\mathrm{C})_{n}=\right)$ are formed after ion 
bombardment of acetylene $\left(\mathrm{C}_{2} \mathrm{H}_{2}\right)$, ethylene $\left(\mathrm{C}_{2} \mathrm{H}_{4}\right)$, ethane $\left(\mathrm{C}_{2} \mathrm{H}_{6}\right)$, and benzene $\left(\mathrm{C}_{6} \mathrm{H}_{6}\right)$. Jones \& Kaiser (2013) have studied the effects of electron irradiation of pure $\mathrm{CH}_{4}$ by reflectron time-offlight mass spectrometry and have found that high-molecular-weight hydrocarbons of up to $\mathrm{C}_{22}$, among them alkanes, alkenes and alkynes, are formed. Other experiments (e.g. Kobayashi et al. 1995; Munõz-Caro et al. 2002; Bernstein et al. 2002; Holtom et al. 2005) have shown, by high performance liquid chromatography, that amino acid precursors are present in the residue formed after energetic processing of simple ice mixtures.

Based on these experimental results it has been suggested that icy grain mantles are constituted not only by the molecular species that have been firmly identified but also by other, more complex, molecules which cannot be detected in the solid phase by IR spectroscopy. These species are expected to enrich the gas phase composition after desorption of icy grain mantles (e.g. Palumbo et al. 2008; Modica \& Palumbo 2010) and could be incorporated in planetesimals and comets.

Finally, these results support the experimental effort (e.g. Allodi et al. 2013) to use more sensitive techniques to evidence the formation of complex molecules and/or fragments that could be of primary relevance for Astrobiology also to understand which species should be searched for, by ground-based or space-born facilities, in protostellar environments, protoplanetary disks and in the atmospheres of extrasolar planets and moons.

\section{Why SKA?}

The numerous detections of high-excitation COM lines in the mm-window call for observations at lower wavelengths, where heavy species are expected to emit (JPL, http://spec.jpl.nasa.gov, and CDMS, http://www.astro.uni-koeln.de/cdms). Even more important, the millimeter frequency bands are often so full of lines that it is paradoxically difficult to identify species through a large number of emission lines simply due to confusion. On the other hand, this low frequency band is relatively clear, given that low energy transitions of light molecules fall at much higher frequencies. The completion of the results obtained at mm-wavelengths with spectral surveys in the cm-window will allow one to have the possibility to have, for different species, a large number of transitions, which is needed to reliably detect the largest COMs for which the population is distributed over many energy states, having large partition functions. Figure 8 shows as an example the simulation of the $\mathrm{HCOOCH}_{3}$ and $\mathrm{HCOCH}_{2} \mathrm{OH}$ spectrum as expected for SKA1-MID modeled with GILDASWeeds (Maret et al. 2011) in the range $\sim 1-15 \mathrm{GHz}$, assuming LTE conditions and physical parameters expected in hot-corinos (see e.g. Jörgensen et al. 2012). In particular, if we consider bright lines $\left(\mathrm{S} \mu^{2} \geq 1 \mathrm{D}^{2}\right)$ at low excitation $\left(\mathrm{E}_{u} \leq 20 \mathrm{~K}\right)$, then the $1-15 \mathrm{GHz}$ band contains a considerable number of COMs transitions. As an example the line densities for bright, low-excitation transitions of methyl formate and glycoaldehyde in the $1-15 \mathrm{GHz}$ range is $\sim 1$ line/GHz, ten times higher than in the 80-300 GHz spectral window.

An instructive view is given by the NRAO 100-m GBT PRIMOS Legacy Project, which recorded the spectrum from $300 \mathrm{MHz}$ to $46 \mathrm{GHz}$ towards the Sgr B2(N) molecular cloud. The PRIMOS data have resulted in numerous new detections in astrochemistry (e.g. Loomis et al. 2013, and references therein). The COM emission is expected to come from regions at subarcsec scale (hot corinos, jets, shocks). SKA1 with band 5 operating at 5-14 GHz will reach $\sim 35$ mas resolution. Therefore, the spatial resolutions offered by SKA are fundamental to re- 


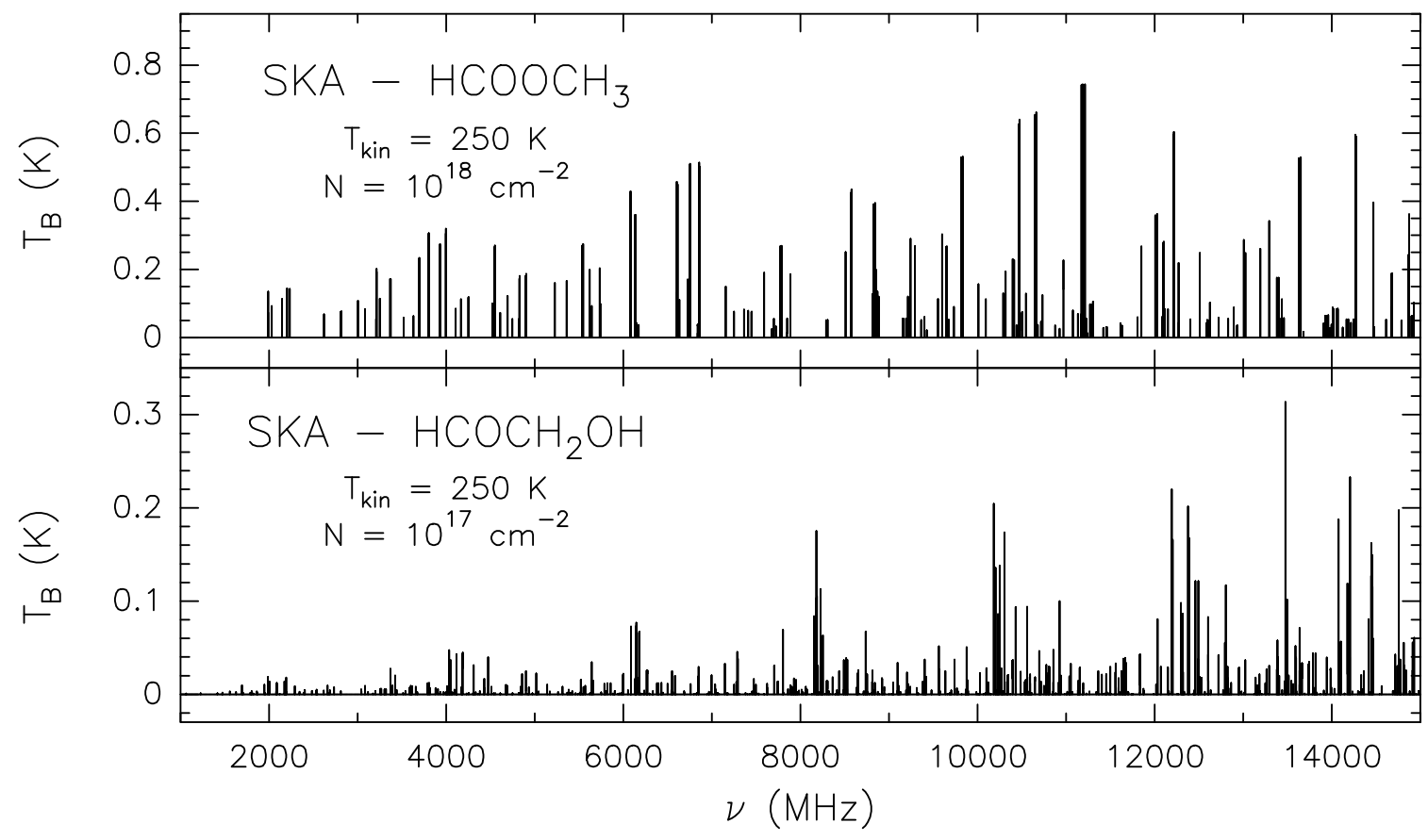

Figure 8: Simulations of the spectrum of $\mathrm{HCOOCH}_{3}$ and $\mathrm{HCOCH}_{2} \mathrm{OH}$ as observed by SKA1-MID modeled with GILDAS-Weeds (Maret et al. 2011) in the range $\sim 1-15 \mathrm{GHz}$, assuming LTE conditions and physical parameters expected for methyl formate and glycolaldehyde in hot-corinos (see e.g. Jörgensen et al. 2012): $T_{\text {kin }}=250 \mathrm{~K}, N\left(\mathrm{HCOOCH}_{3}\right)=10^{18} \mathrm{~cm}^{-2}, N\left(\mathrm{HCOCH}_{2} \mathrm{OH}\right)=10^{17} \mathrm{~cm}^{-2}$, source size $=1 "$, and line FWHM $=4 \mathrm{~km} \mathrm{~s}^{-1}$.

solve and image the emitting region. As a comparison, the NRAO VLA interferometer, with $36 \mathrm{~km}$ maximum baseline, obtains a synthetisized beam between 130 mas (15 GHz) and 2" (1 $\mathrm{GHz}$ ) (https://science.nrao.edu/facilities/vla/docs/manuals/oss). In this way, (i) we will have bright line emission, and (ii) we will correctly evaluate COMs' abundances. In addition, the sensitivity achieved by SKA for larger beam sizes will be unbeatable, offering the possibility to detect the emission from glycine and other amino acids in pre-stellar cores, where their emission is expected to be relatively extended (around tens of arcseconds). To conclude, SKA offers a unique combination of spatial resolution and high sensitivity to produce a complete inventory of known interstellar species accessible in the centimeter wavelength range that can be used to put severe constrains on the physical conditions of the emitting gas, as well as on COMs abundances.

This work was partly supported by the Italian Ministero dell'Istruzione, Università e Ricerca (MIUR) through the grant Progetti Premiali 2012 - iALMA.

\section{REFERENCES}

Allodi, M.A., Baragiola, R.A., Baratta, G.A., et al. 2013, Space Science Reviews 180, 101

Baratta, G.A., Leto, G., Palumbo, M.E. 2002, A\&A 384, 343

Bennett, C.J., Chen, S.-H., Sun, B.-J., Chang, A.H.H., Kaiser, R. 2007, ApJ 660, 1588

Arce, H.G., Santiago-García, J., Jörgensen, J.K., Tafalla, M., Bachiller, R. 2008, A\&A 681, L21 
Bachiller, R., Pérez Gutiérrez, M., Kumar, M.S.N., Tafalla, M. 2001, A\&A 372, 899

Bacmann, A., Taquet, V., Faure, A., Kahane, C., Ceccarelli, C. 2012, A\&A 541, L12

Belloche, A., Müller, H.S.P., Menten, K.M., Schilke, P., Comito, C. 2013, A\&A 559A, 47B

Belloche, A., Menten, K.M., Comito, C., et al. 2008, A\&A 482, 179

Beltrán, M.T., Codella, C., Viti, S., Neri, R., Cesaroni, R. 2009, ApJ, L93

Benedettini, M., Viti S., Codella, C., et al. 2007, MNRAS 381, 1127

Benedettini, M., Busquet, B., Lefloch, B., et al. 2012, A\&A 539, L3

Bernstein, M.P., Dworkin, J.P., Sandford, S.A., Cooper, G.W., Allamandola, L.J. 2002, Nature 416, 401

Bisschop, S.E., Jörgensen, J.K., Bourke, T.L., Bottinelli, S., van Dishoeck, E.F. 2008, A\&A 488, 959

Bottinelli, S., Ceccarelli, C., Williams, J.P., Lefloch, B. 2007, A\&A 463, 601

Busquet, B., Lefloch, B., Benedettini, M., et al. 2014, A\&A 561, 120

Calcutt H., Viti S., Codella C., et al. 2014, MNRAS, in press

Caselli P., Ceccarelli C., 2012 The Astronomy and Astrophysics Review, 20, 56

Caselli, P., Walmsley, C.M., Tafalla, M., Dore, L., Myers, P.C. 1999, ApJ 523, L165

Caselli, P., Keto, E., Bergin, E.A., et al. 2012, ApJ 759, L37

Caselli, P., Walmsley, C.M., Tafalla, M., Dore, L., Myers, P.C. 1999, ApJL, 523, L165

Cazaux, S., Tielens, A.G.G.M., Ceccarelli C., et al. 2003, ApJ 593, L51

Ceccarelli, C., Caselli, P., Herbst, E., Tielens, A.G.G.M., Caux, E. 2007 Protostars and Planets V, University of Arizona Press, Tucson, 47

Ceccarelli, C., Bacmann, A., Boogert, A., et al. 2010, A\&A 521, L22 Protostars and Planets V, University of Arizona Press, Tucson, 197

Codella, C., Benedettini, M., Beltrán, M.T., et al. 2009, A\&A 507, L25

Codella, C., Fontani, F., Ceccarelli, C., et al. 2014, ApJL, submitted

Compagnini, G., D’Urso, L., Puglisi, O., Baratta, G. A., Strazzulla, G. 2009, Carbon 47, 1605

Crapsi, A., Caselli, P., Walmsley, C.M., Tafalla, M. 2007, A\&A 470, 221

Cuppen, H.M., van Dishoeck, E.F., Herbst, E., Tielens, A.G.G.M. 2009, A\&A 508, 275

Ehrenfreund, P., Kerkhof, O., Schutte, W.A. et al. 1999, A\&A 350, 240

Ehrenfreund, P., Glavin, D.P., Botta, O., Cooper, G., Bada, J.L. 2001, PNAS 98, 2138

Elsila, J.E., Glavin, D.P., Dworkin, J.P. 2009, M\&PS 44, 1323

Fontani, F., Palau, A., Caselli, P., et al. 2011, A\&A 529, L11

Fontani, F., Giannetti, A., Beltrán, M.T., et al. 2012, MNRAS 423, 2342

Fontani, F., Codella, C., Ceccarelli, C., Lefloch, B., Viti, S., Benedettini, M. 2014a, ApJ 788, L43

Fontani, F., Busquet, G., Palau, A., et al. 2014b, A\&A, in press

Foti, G., Calcagno, L., Sheng, K.L., Strazzulla, G. 1984, Nature 310, 126

Friesen, R.K., Di Francesco, J., Shimajiri, Y., Takakuwa, S. 2010, ApJ 708, 1002

Fuente A., Cernicharo J., Caselli P., et al. 2014, A\&A 568, 65

Gibb, E.L., Whittet, D.C.B., Boogert, A.C.A., Tielens, A.G.G.M. 2004, ApJS 151, 35

Glavin, D.P., Dworkin, J.P., Aubrey, A., et al. 2006, M\&PS 41, 889

Grim, R.J.A., \& Greenberg, J.M. 1987, ApJ 321, L91

Gueth F., Guilloteau S., \& Bachiller R. 1996, A\&A 307, 891

Gueth F., Guilloteau S., \& Bachiller R. 1998, A\&A 333, 287 
Herbst, E., van Dishoeck, E.F. 2009, ARA\&A 47, 427

Holtom, P.D., Bennett, C.J., Osamura, Y., Mason, N.J., Kaiser, R.I. 2005, ApJ 626, 940

Hudson, R.J., \& Moore, M.H. 2000, Icarus 145, 661

Hudson, R.L., Moore, M.H., Gerakines, P.A. 2001, ApJ 550, 1140

Islam, F., Baratta, G.A., Palumbo, M.E. 2014, A\&A 561, A73

Jiménez-Serra, I., Testi, L., Caselli, P., Viti, S. 2014, ApJ 787, L83

Jones, B.M., \& Kaiser R.I. 2013, J. Phys. Chem. Lett. 4, 1965

Jörgensen, J.K., Favre, C. Bisschop, S.E., Bourke, T.L., van Dishoeck, E.F., Schmalzl, M. 2012, ApJ 757, L4

Kahane, C., Ceccarelli C., Faure, A., Caux, E. 2013, ApJ 763, L38

Kobayashi, K., Kasamatsu, T., Kaneko, T., et al. 1995, Adv. Space Res. 16, 21

Kurtz, S., Cesaroni, R., Churchwell, E., Hofner, P., Walmsley, C.M. 2000 Protostars and Planets IV, University of Arizona Press, Tucson, 299

Loomis, R.A., Zaleski, D.P.; Steber, A.L., et al. 2013, ApJ 765, L9

López-Sepulcre, A., Mendoza, E., Lefloch, B., et al. 2014, MNRAS, submitted

Marcelino, N., Cernicharo, J.; Agúndez, M., et al. 2007, ApJ 665, L127

Maret S., Hily-Blant P., Pety J., et al., A\&A 526, A47

Maury, A.J., Belloche, A., André, Ph., et al. 2014, A\&A 563, L2

Mendoza, E., Lefloch, B., López-Sepulcre, A., et al. 2014, MNRAS 445, 151

Modica, P., Palumbo, M. E., 2010, A\&A 519, 22

Moore, M.H., Donn, B., Khanna, R., \& A'Hearn, M. 1983, Icarus, 54, 388

Moore, M. H., Ferrante, R. F., Nuth J. A. 1996, Planetary and Space Science, 44, 927

Muñoz Caro, G.M., Meierhenrich, U.J.; Schutte, W.A., et al. 2002, Nature 416, 403

Neufeld, D.A., Nisini, B., Giannini, T., et al. 2009, ApJ 706, 170

Öberg, K.I., Fraser, H.J., Boogert, A.C.A., et al. 2007, A\&A, 462, 1187

Öberg, K.I., Garrod, R.T., van Dishoeck, E.F., Linnartz, H., 2009, A\&A 504, 891

Palumbo, M.E., \& Strazzulla, G. 1993, A\&A 269, 568

Palumbo, M.E., Castorina, A.C., Strazzulla, G. 1999, A\&A 342, 551

Palumbo, M.E., \& Baratta, G.A. 2000, A\&A 361, 298

Palumbo, M.E., Ferini, G., Baratta, G.A. 2004, Advances in Space Research 33, 49

Palumbo, M.E. 2006, A\&A 453, 903

Palumbo, M.E., Leto, P., Siringo, C., Trigilio, C. 2008, ApJ 685, 1033

Parise, B., Castets, A., Herbst, E., et al. 2004, A\&A 416, 159

Parise, B., Ceccarelli, C., Tielens, A.G.G.M., et al. 2006, A\&A 453, 949

Peng, T.-C., Despois, D., Brouillet, N., Parise, B., Baudry, A. 2012, A\&A 543, 152

Pizzarello, S., Krishnamurthy, R.V., Epstein, S., Cronin, J.R. 1991, GeCoA 55, 905

Puglisi, O., Compagnini, G., D’Urso, L., Baratta, G.A., Palumbo, M.E., Strazzulla, G. 2014, Nucl. Instr. Meth. B. 326, 2

Raut, U., Teolis, B.D., Loeffler, M.J., Vidal, R.A., Famá, M., Baragiola, R.A. 2007, J. Chem. Phys. 126,244511

Sandford, S.A., Allamandola, L.J., Tielens, A.G.G.M., Valero, G.C. 1988, ApJ 329, 498

Strazzulla, G., Baratta, G.A., Palumbo, M.E. 2001, Spectroch. Acta A 57, 825

Tafalla M., \& Bachiller R. 1995, ApJ 443, L37 
Taquet, V., Ceccarelli, C., Kahane, C. 2012, A\&A 538, 42

Testi, L., Perez, L., Jimenez-Serra, I., et al., 2015, Protoplanetary disks and the dawn of planets with SKA, in proceedings of "Advancing Astrophysics with the Square Kilometre Array", PoS(AASKA14) 117 